\title{
The Carbon-Water Interface: Opportunities for the Water-Energy Nexus
}

\author{
Alberto Striolo \\ Department of Chemical Engineering \\ University College London, Torrington Place, London WC1E 7JE, UK \\ a.striolo@ucl.ac.uk
}

\section{Angelos Michaelides}

Thomas Young Centre, London Centre for Nanotechnology, and Department of Chemistry, University College London, 17-19 Gordon Street, London WC1H 0AH, UK angelos.michaelides@ucl.ac.uk

\section{Laurent Joly}

Institut Lumière Matière, UMR5306 Université Lyon 1-CNRS, Université de Lyon 69622 Villeurbanne, France

laurent.joly@univ-lyon1.fr

Providing clean water and sufficient affordable energy to all without compromising the environment are key priorities of the scientific community. Many recent studies have focused on carbon-based devices in the hope of addressing these grand challenges, justifying and motivating detailed studies of water in contact with carbonaceous materials. Such studies are becoming increasingly important because of the miniaturization of newly proposed devices, with ubiquitous nano-pores, large surface-to-volume ratio, and many, perhaps most of the water molecules at contact with a carbon-based surface. In this brief review we discuss some recent advances obtained by simulations and experiments in the development of carbon-based materials for applications in water desalination. We suggest possible ways forward, with particular emphasis on the synergistic combination of experiments and simulations, with simulations now sometimes offering sufficient accuracy to provide fundamental insights. We also point the interested reader to recent works that complement our short summary on the state of the art of this important and fascinating field. 


\section{Introduction: the importance of reverse osmosis in water desalination}

The scientific community is facing the challenge of providing technological solutions for the water-energy nexus while preserving the environment. Carbon-based devices have been developed both for desalinating water and for storing energy. Such devices are characterized by vast carbon-water interfaces, due to the ubiquity of nano-pores. It is indeed possible that many, perhaps most water molecules within such devices come in contact with the carbon surface. Hence, a detailed understanding of water-carbon interfaces is necessary to ensure progress. Here, we review some recent advances obtained by atomistic simulations and experiments on this field.

Traditional water desalination approaches such as multi-flash distillations are widely used, but require large amounts of energy. As such, they are not sustainable in the long run. Reverse osmosis (RO) has become the technology of choice for most new installations. Two innovations are considered to be responsible for the wide application of RO. The first was the introduction of thin film composite membranes; the other was the implementation of energy recovery devices that re-use part of the energy present in pressurized brine (i.e., pressure exchangers and efficient pumps) $(1,2)$. Although RO is mature and reliable, it remains energy intensive (3-5). At its core are membranes permeable to water and impermeable to salt. The state-of-the art membranes, based on polyamide thin film composites, degrade in the presence of chlorine (which makes disinfection difficult) and are prone to fouling (6). Many believe that 'in order for desalination to live up to the water challenges of the $21^{\text {st }}$ century a step-change is needed in RO membrane technology' (7). Although high mechanical strength as well as resistance to degradation and fouling are prerequisites to practical applications, much fundamental research seeks to identify nano-structured membranes that could yield high water fluxes and high salt rejection at the expense of limited pressure drops.

The remainder of this article is structured as follows: we first discuss some recent work related to the development of nano-structured membranes for water desalination, based either on carbon nanotubes or on graphene; we then propose a few ways forward, which we think could lead to the improvement of RO, as well as one alternative water desalination process (capacitive deionization); we then discuss how computer simulations could develop to continue to be relevant in this field. Rather than a comprehensive review, this is a brief personal view on the subject. For other complementary perspectives we refer the reader to previous discussions of emerging desalination technologies $(5,8-12)$ and nanofluidic transport (13-18). 

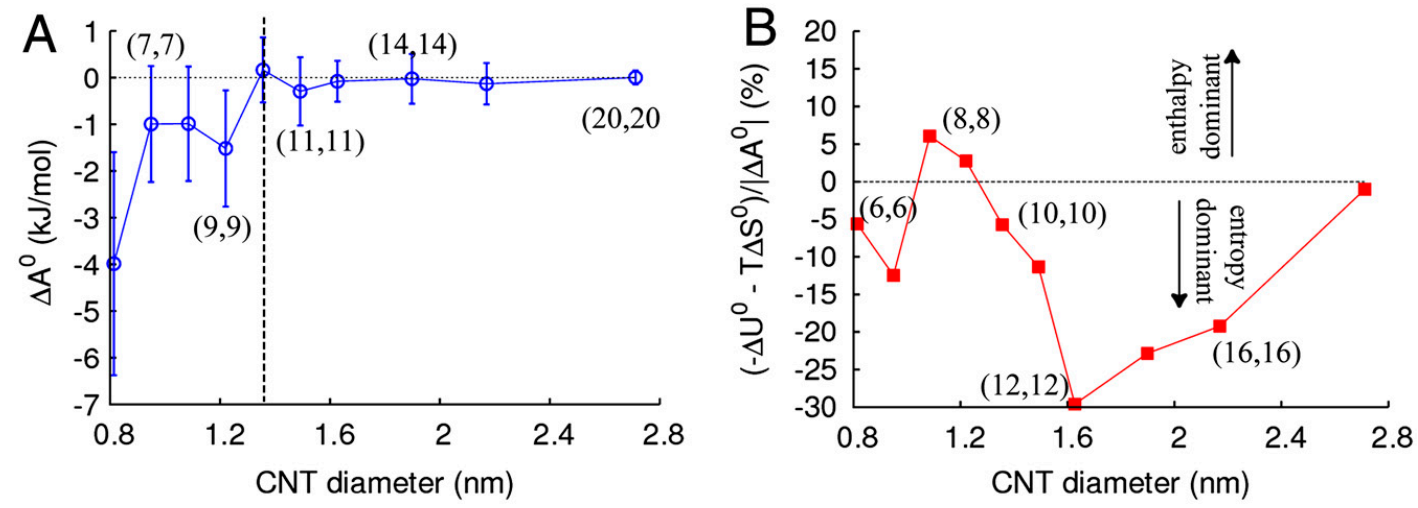

Figure 1. Changes in free energy for water molecules as they transfer from bulk liquid to the interior of infinite single-walled carbon nanotubes of varying diameter: simulations conducted at ambient conditions with the SPC/E model for water. The CNTs are labeled according to their chirality. Panel A: Relative Helmholtz free energy, $\Delta \mathrm{A}^{0}$ (confined water relative to bulk water). The dashed line indicates the convergence for bulk water. Panel B: Percentage of the free energy $\Delta \mathrm{A}^{0}$ arising from enthalpic $\left(\Delta \mathrm{U}^{0}\right)$ or entropic contributions $\left(\Delta \mathrm{S}^{0}\right)$. The dashed line indicates conditions at which the two contributions cancel each other out. Figure reproduced from Ref. (19).

\section{Carbon nanotube - based membranes}

Among membranes likely to improve RO performance, those based on carbon nanotubes (CNTs) have attracted significant attention since early simulations showed that water molecules can travel extremely quickly through narrow CNTs (20), suggesting low drag losses (21). Considerable progress has been made, with experimental data in general consistent with the early simulations. Because CNTs are made of hydrophobic sp2-hydridized carbon atoms, the initial simulations reporting water adsorption inside CNTs attracted attention - sometimes even skepticism. With a view to understanding this puzzling result, the free energy changes for water as it enters CNTs of various diameters at ambient conditions were recently computed (19). The results show that the classical atomistic model of water known as SPC/E adsorbs spontaneously within CNTs of diameter in the range from 0.8 to $2.7 \mathrm{~nm}$, but that the driving force depends on diameter (see Figure 1). For 0.8-1.0 nm CNTs, confined water is in a vapor-like phase stabilized by entropy. Whereas water confined within 1.1-1.2 nm CNTs is ice-like, stabilized by enthalpy. Water confined in CNTs wider than $1.4 \mathrm{~nm}$ is liquid, in a bulk-like structure stabilized by large translational entropy. Many simulations also considered the transport of water inside CNTs (22). Among these, Striolo used molecular dynamics (MD) simulations to show that the fast water transport is due to the combination of strong water-water attractions and relatively weak and smooth CNT-water interactions (23). Falk et al. $(24,25)$ later identified the crucial role of wall curvature in reducing water friction at the wall of CNTs. Joseph and Aluru investigated the pressure-driven flow of liquid water through $(16,16)$ CNTs of diameter $2.17 \mathrm{~nm}$ (26). They found a $\sim 2000$ times enhancement of mass flow rate with respect to that predicted by continuum theory, a consequence of the velocity 
'jump' in a region depleted of water at the water-CNT interface. Joseph and Aluru also showed that changes in the effective tube-water interactions, atomic-scale roughness of the CNTs walls, and even small increases in the atomic carbon-carbon distance within the CNTs can decrease the water flow, further suggesting that the nanotube properties can manipulate the water transport. As one might expect, even just a few defects, e.g., a few oxygenated sites (27), or constrictions along the tube axis (28), can affect the water flow through CNTs.

On the experimental front, Majumder et al. $(29,30)$ fabricated membranes composed of aligned multi-walled CNTs of $\sim 7 \mathrm{~nm}$ diameter, with area density of $\sim 5 \cdot 10^{10}$ per $\mathrm{cm}^{2}$, crossing a polystyrene film. They measured water permeability, and they estimated the fluid velocities through the CNTs to be 4-5 orders of magnitude faster than those predicted by the Hagen-Poiseuille equation assuming laminar flow and zero velocity at the pore walls. They estimated a slip length from $\sim 3$ to $70 \mu \mathrm{m}$, much larger than the pore radius, and they reported that the fast water flow decreased after a few minutes. The interpretation of the fast water transport was consistent with the simulations discussed above (although the measured slip lengths were more than 2 orders of magnitude larger than the most favorable MD prediction (14)), while the decrease of flow over time was ascribed to a flowinduced solvent ordering. It was pointed out that the availability of a high density of pores allows for macroscopic measurements of fluid transport. However, Sisan and Lichter suggested that difficulties in accurately determining channel radii, the number of channels spanning the membrane, and perhaps in determining the net flow rates could yield inaccurate estimates of water flow rates through CNT membranes (31). In fact, when the pore properties are measured, different data for fluid transport are obtained. Using membranes with mono-disperse CNTs of inner diameter $\sim 2 \mathrm{~nm}$, Holt et al. (32) reported water flow rates 2-4 orders of magnitude faster than those predicted by continuum theories, but a few orders of magnitude smaller than those reported by Majumder et al. (30), and consistent with MD predictions (14). Experiments on individual CNTs of $0.81-1.59 \mathrm{~nm}$ diameter showed flow enhancement rates below 1000 (33), and sometimes just modest enhancements of water transport through individual CNTs (34). In summary, the experimental data are consistent in showing enhanced water flow through narrow CNTs, but the enhancement factor with respect to continuum fluid dynamics calculations is not uniquely determined.

One would expect that molecular simulations would help to identify not only the mechanism by which flow is enhanced, but also to quantify the enhancement factor. Unfortunately, simulations have not been conclusive. We will discuss below some of the inherent limitations in the accuracy of the classical models used in typical waterCNT simulations. However, here we comment on the fact that very often simulations are conducted in periodic systems. In such cases potentially important poreentrance and pore-exit effects are ignored (31). Indeed, Joly highlighted the crucial role of entrance effects in the capillary filling of a CNT with water using MD (35), and Gravelle et al. $(36,37)$ showed that using a conical entrance could minimize 
entrance dissipation. Walther et al. (38) conducted massive MD simulations to reproduce the experimental set up of Holt et al. (32) (i.e., double-walled CNTs spanning hydrophilic membranes 3 - $2000 \mathrm{~nm}$ thick). Three stages were identified: (a) water entry and filling of the CNTs; (b) water emergence and droplet formation at the pore exit; and (c) water flow through the CNT connecting two water reservoirs. Under imposed pressures of $\sim 1$ bar, water entry and exit from the CNTs depended on the state of the membranes, with pre-wetted membranes allowing for water to infiltrate the CNTs. Data analysis showed a slip length of $\sim 63 \mathrm{~nm}$, independent on the thickness of the membrane, and a significant entrance loss. Entrance losses were found to be the dominant losses for membranes of thickness 3 $\mathrm{nm}$ or less. Once the entry losses are accounted for, Walther et al. (38) showed that the flow rate increases linearly with the pressure gradient, which was not in agreement with Thomas and McGaughey (39), who reported nonlinear dependence but did not account for entrance losses. Walther et al. (38) estimated that entrance and exit effects could be ignored for CNT-based membranes with thickness of at least $\sim 300 \mathrm{~nm}$. It is possible that functionalizing the entrance of the CNT affects these resistances: Majumder et al. (40) functionalized the CNTs ends in their membranes and reduced water permeability compared to un-functionalized CNTs by 3 orders of magnitude; when they functionalized also the interior of the CNTs the increase in water flux was only a factor of 5 compared to traditional expectations (30). The interpretation proposed for the experimental observations is that the functional groups affect the slip boundary condition.

We concentrate now on salt rejection. Thomas et al. (16) proposed six mechanisms to tailor salt rejection in nanoporous membranes: (a) size exclusion of the bare ion; (b) steric exclusion of the hydrated ion; (c) charge repulsion; (d) specific ion-pore interactions; ( $\mathrm{e}$ ) interactions between ions and chemical structures in the pore; and (f) entropic differences. By manipulating the CNT diameter, it is possible to maximize the first three of these criteria. The fourth criterion refers to subtle effects due to the overall morphology of the pore, as suggested by biological pores $(41,42)$. A study inspired by structure-function properties of biological channels showed that it is possible to control the conduction of ions through a CNT compressed laterally (28). These simulations were conducted for aqueous electrolyte solutions containing $\mathrm{KCl}$ through $(12,12)$ CNTs. Water, $\mathrm{K}^{+}$and $\mathrm{Cl}^{-}$ions were able to flow through a pristine $(12,12)$ CNT; when the central section of the CNT was compressed to form a conical constriction, first the ions were not allowed to flow through, and then neither water nor ions flowed. The blockage to ionic fluxes was due to the dehydration of the ions. This simulation suggests the possibility of tuning the conductivity of nano-structured membranes using mechanical deformations, which are probably reversible because of the CNTs' materials properties.

\section{Graphene - based membranes}

Because of graphene's mechanical strength, its imperviousness to atoms as small as helium, and optimistic atomistic simulation results (43-45), it has been predicted that 'nanoporous graphene with a high density of subnanometer pores' could yield 'ultrafast water permeance and high salt rejection because the atomic thinness 
provides little resistance to flow, yet blocks the passage of solutes that are larger than the pores' (46). Note however that Gravelle et al. showed, using MD simulations, that the permeability of a small CNT with conical entrances can exceed that of a graphene sheet pierced with a hole of similar size to the CNT diameter (37). CohenTanugi and Grossman reviewed computational studies on single-layer graphene for RO (7).

Assuming a pore density of $\sim 10^{13}$ nanopores per $\mathrm{cm}^{2}$, Cohen-Tanugi and Grossman, using non-equilibrium MD simulations, estimated a permeability $c a$. 2-3 orders of magnitude larger than that observed in thin film composite membranes. The results depended greatly on the nanopore size, and on the functional groups at the pore edges. The pores needed to be $c a .0 .27 \mathrm{~nm}$ to reject salt effectively. Because the efficiency falls dramatically as the pore size increases to $\sim 0.4 \mathrm{~nm}$, to manufacture RO membranes it is necessary to achieve extremely high accuracy in producing pores, and to ensure that the graphene sheets remain intact during operation. Konatham et al. (47) investigated, using equilibrium MD, how functionalizing the pores could help with rejecting salt ions. They reported the effective potential of mean force (PMF) for water, $\mathrm{Na}^{+}$, and $\mathrm{Cl}^{-}$ions through the pore. The narrow pores were decorated with chemical groups such as $\mathrm{COO}^{-}$and $\mathrm{OH}$. The PMF was calculated along the vertical distance from graphene, through the pore center. High free energy barriers when the molecules approached the pore were interpreted as signatures of effective rejection (i.e., high water permeability requires small free energy barriers). The results showed that if the pores are too narrow, steric effects prevent water from flowing through; if the pores are too wide, the functional groups are not sufficient to reject salt. When the pores are $\sim 0.75 \mathrm{~nm}$ in diameter, functional groups can help: charge effects are effective (i.e., COO- groups yield high free energy barriers for $\mathrm{Cl}^{-}$ions), but as the salt concentration increases, electrostatic repulsions are screened. It was encouraging to observe that $-\mathrm{OH}$ groups remained effective at rejecting both $\mathrm{Na}^{+}$and $\mathrm{Cl}-$ ions at $0.25 \mathrm{M}$. These results suggest that steric effects remain a dominant mechanism for salt exclusion at moderate salt concentrations.

Despite encouraging computational results, leakage has prevented the implementation of graphene-based RO devices. To address this limitation O'Hern et al. (48) produced a graphene layer and transferred it onto a porous polycarbonate membrane that provides structural support. The graphene shows $1-15 \mathrm{~nm}$ pores and large tears. The Authors filled the tears using atomic layer deposition and repaired the nanometer-scale defects using Nylon 6,6. New pores of size $0.5 \mathrm{~nm}$ were introduced by ion bombardment, followed by etching (48). The membrane permeability was comparable to that of current RO membranes for seawater desalination (49). The membrane was tested with respect to the rejection of dextran (Mw $4.4 \mathrm{kDa}$ ), Allura Red (size $\sim 1 \mathrm{~nm}$ ), $\mathrm{MgSO}_{4}$, and $\mathrm{NaCl}$. The results were encouraging for dextran, Allura Red, and $\mathrm{MgSO}_{4}$. Unfortunately, $\mathrm{NaCl}$ was found to permeate the membrane, possibly because it is able to diffuse through the Nylon 6,6 plugs. Because of the technical limitations just mentioned, and also because of the difficulty in generating pores well-controlled in size and chemical functionalization, it has so far only been possible to conduct extensive experimentation on single-layer 
graphene membranes of microscopic areas with a few pores (50-53). 0'Hern et al. (48) reported the synthesis of single-layer graphene membranes with pores that are uniform in size and whose chemical functionalization can be tailored. XPS measurements suggested the pores were terminated with ketone, quinone, hydroxyl, and/or carbonyl groups. The resultant membranes were tested towards their efficiency at repelling aqueous $\mathrm{KCl}$ and Allura Red. The permeability of both compounds increased using etching for 5 minutes, and if etching continued for longer than 25 minutes the membranes only rejected Allura Red, as the pores became too large to regulate ion transport. While the membranes were not strong enough to be tested in pressure-driven RO experiments, the results suggest, in qualitative agreement with simulations, that it is possible to tune the pore properties, and doing so allows for the performance of graphene-based membranes to be regulated.

As an alternative to single-layer graphene, many researchers are considering stacks of multiple graphene layers, after Nair et al. (54) reported that graphene oxide (G0) membranes can be impermeable to helium and other gases, while allowing the 'unimpeded permeation of water'. GO membranes are easy to fabricate and mechanically robust, suggesting the possibility of large scale production $(55,56)$. Their performance however depends on the preparation, as demonstrated by Kim et al. (57). This group showed that selectivity to gas transport can be achieved by controlling the degree of interlocking between the various GO sheets. Interlocking is expected to change the flow channels and the pore sizes. GO laminates were proposed for several applications, including $\mathrm{CO}_{2}$ separation from gases (58) and RO $(59,60)$, and have been used for pressure-driven separations (60-63). Joshi et al. (64) reported on membranes prepared from GO suspensions using vacuum filtration. The manufactured membranes were vacuum-tight in the dry state, but showed significant water flow rates when exposed to liquid aqueous solutions. Using different experimental techniques, the Authors found that cations and ions moved through the membranes in stoichiometric amounts to maintain charge neutrality, and that the permeation rates were a strong function of the size of the hydrated ions, with species larger than $\sim 0.45 \mathrm{~nm}$ effectively sieved out of the membrane. Atomistic MD simulations of graphene capillaries of width sufficient to allow the formation of 1,2 , and 3 layers of water molecules support their interpretation. The Authors found that when the width of the pore allowed the formation of a single layer of water, no ions were able to penetrate the capillaries; the ions were able to penetrate the pores when 2-3 water layers were present, but any molecule larger than $\sim 0.45 \mathrm{~nm}$ was excluded. The combined simulation/experiment results suggest that the permeation of the GO membranes is determined by regions within the pore network in which only 2-3 layers of water molecules are allowed. Therefore understanding structure and dynamics of aqueous solutions confined in narrow carbon-based pores is of tremendous importance. Many studies have been concerned with the adsorption and transport of water in carbon-based porous materials (65-67). For example, Striolo et al. (68) investigated systematically the adsorption isotherms for water in carbon-slit pores of varying widths, in a wide temperature range. In qualitative agreement with experiments, it 
was proposed that the hysteresis critical temperature depends on the pore width, and also on the pores shape (69).

For the scope of the present review, it is more important to point out how the simulation results help identify the structure of confined liquid water, often quantified in terms of density profiles. See, for example, in Figure $\mathbf{2}$ how the pore width affects the layers of water. Understanding water confined in slit-shaped carbon pores is also relevant in understanding hydrophobic interactions (70-73). The length scale at which liquid water becomes unstable when confined between hydrophobic plates has recently been quantified (74-76), as well as the molecular pathways that lead to de-wetting (77). Bakli and Chakraborty used MD to assess whether the presence of salts affects the hydrodynamics of water confined in slit channels (78), with the water-solid interactions chosen to reproduce wetting and non-wetting conditions (monitored by the sessile contact angle). The Authors observed a direct relationship between contact angle and slip length, with no slip in the wetting channel, and some slip in the non-wetting one. The Authors then observed that when salt was added to the wetting system, the slip length increased, while when salt was added to the non-wetting system the slip length decreased. The slip length correlated with the maximum in molecular density within the first hydration layer, consistent with the analysis of slip length by Barrat and Bocquet (79) based on the Green-Kubo formalism. Bakli and Chakraborty also found evidence of salt-specific effects related to the ion hydration strength (49), and extended their investigation to electrokinetic energy conversion (80). Joly et al. (81) investigated the effect of salt on slip length with charged confining walls. In that case, the slip length was mainly controlled by the surface charge, while salt concentration effects were negligible.

Several of the studies discussed so far were concerned with the hydrodynamic boundary condition used to describe water flow. Recently, Ho et al. challenged the traditional view according to which hydrodynamic slip can only occur for water on surfaces that are 'hydrophobic' (82). They used non-equilibrium MD to quantify the hydrodynamic slip length on materials that, based on the water contact angle, could be classified as either hydrophobic (graphene) or hydrophilic (materials inspired by magnesium oxide). The results showed evidence for hydrodynamic slip on graphene, as expected (24), but also on some of the hydrophilic materials. The key ingredient for hydrodynamic slip to occur was that the interfacial water molecules should be able to move from one preferential adsorption site to a neighboring one without necessarily desorbing from the first hydration layer. Tocci et al. (83) investigated hydrodynamic slip of liquid water on graphene and, for comparison, a single layer of hexagonal boron nitride using equilibrium ab initio MD (AIMD), with forces obtained 'on the fly' from electronic structure calculations. Green-Kubo relations were used to extract friction coefficients $(84,85)$. It was found that although the structures of the liquid water film on the two surfaces were exceedingly similar, the friction coefficient on boron nitride was $\sim 3$ times larger than that on graphene (see Figure 2), because of the greater corrugation of the energy landscape on boron nitride. This arose from specific electronic structure 
effects and revealed a subtle dependence of the friction on the atomistic details of a surface, which is not directly related to its wetting properties. To ensure progress in GO membranes it should be investigated how oxygenated groups could affect these results, both in terms of the structure and the dynamics of confined water, and in terms of its tendency to de-wet the pores. In fact, it has been shown that the presence of a few groups that favorably interact with water on an otherwise hydrophobic surface strongly affect both the structure of interfacial water $(27,86$, 87 ) and the tendency of water to evacuate the confined region (88-90).
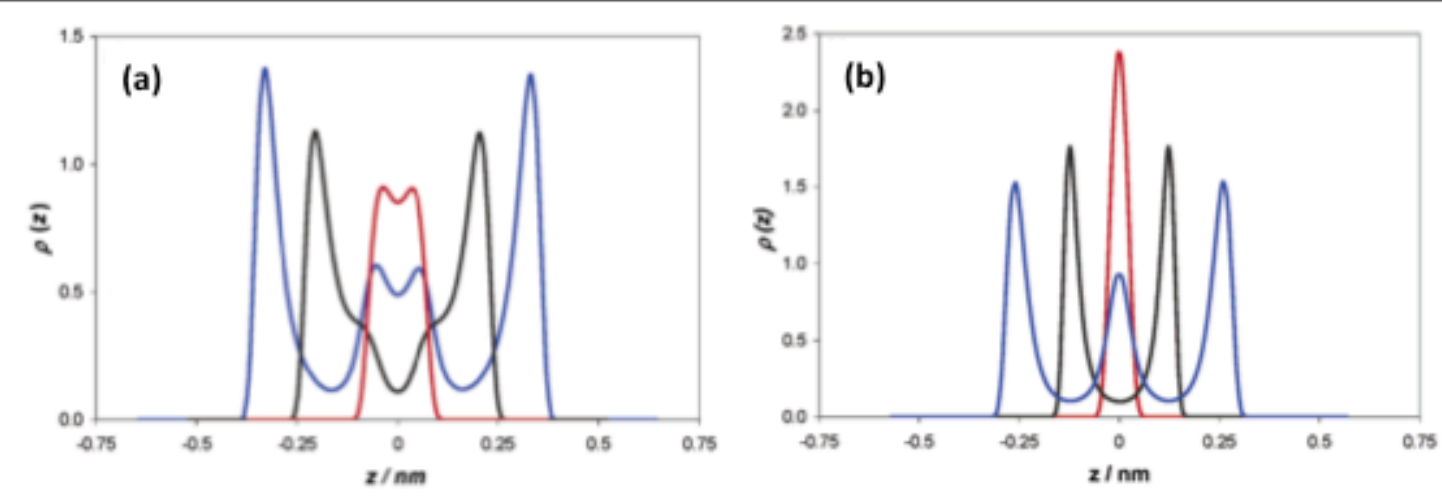

(c)

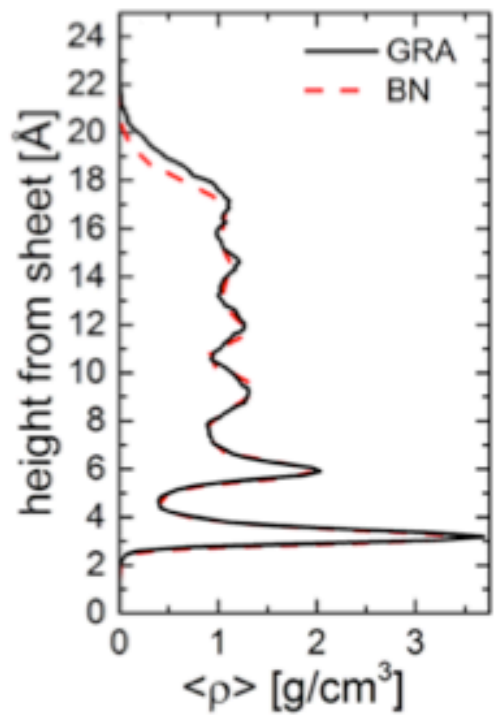

(d)

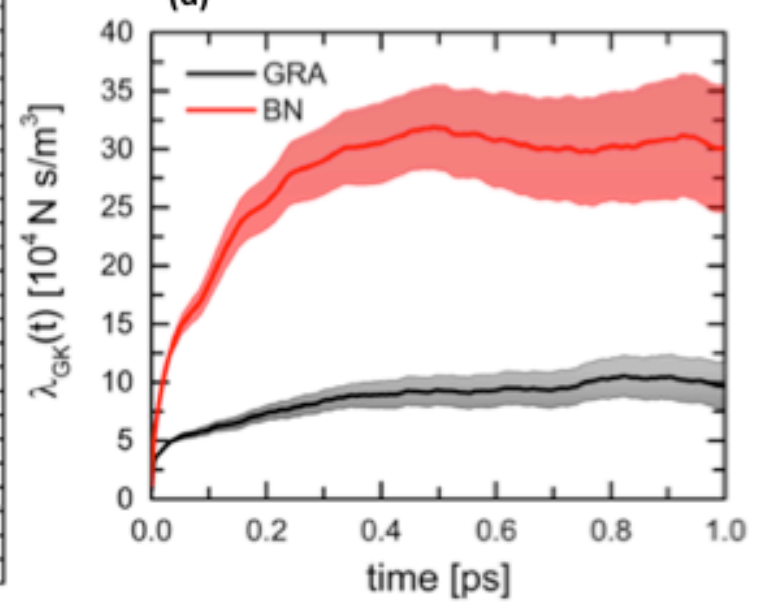

Figure 2. Panels (a) and (b): Density profiles of the oxygen atom of water confined within carbon-slit pores of varying width; these simulations were conducted for the SPC/E model of water, using the grand canonical Monte Carlo algorithm at $298 \mathrm{~K}$ and at conditions at which the pores just filled with liquid water; blue, black, and red lines are for pores of different widths: 1.30, 1.05 and $0.75 \mathrm{~nm}$ in panel (a) and 1.15 , 0.85 , and $0.65 \mathrm{~nm}$ in panel (b), respectively. Panel (c): Structure of a liquid water film on graphene (GRA) and a single sheet of hexagonal BN. Specifically the average density profile $\langle\rho\rangle$ as a function of the height from the sheet is shown. The liquid film structure is essentially the same on both sheets (on BN, the film is slightly thinner because the BN unit cell is slightly larger than that of graphene). Panel (d): Comparison between the Green-Kubo estimate of the friction coefficient of liquid water on graphene and on BN. The shaded areas represent the uncertainties obtained by performing a block average. The friction coefficient $\lambda$ is given by the plateau value at long times. There is an evident increase in the friction coefficient on BN.

Figures adapted from Ref. (68) and Ref. (83). 


\section{Research needs to advance water desalination via reverse osmosis}

To further develop CNT and graphene-based membranes, a synergistic combination of theoretical and experimental studies on single-pore systems is needed. Along these lines, Bocquet and coworkers developed instruments capable of probing extremely small mass transport across individual nano-channels (91) by adapting the Coulter technique (the electrical resistance across a channel increases when a 'large' compound, e.g., a salt ion, crosses the channel because it reduces the amount of water within the channel). Using statistical analysis of measured current as a function of time, they measured water flows with an accuracy of a few femto-liters per second; two orders of magnitude better than state-of-the-art methods. Strano and coworkers investigated individual, isolated CNTs (92). In particular, they monitored the electro-osmotic current through the CNT interior, observing stochastic pore blocking when individual cations partitioned into the CNT. Recently, Strano and coworkers measured, using two complementary techniques, the diameter-dependent liquid-solid phase boundaries for water within CNTs 1.05, 1.06, $1.15,1.24$ and $1.52 \mathrm{~nm}$ in diameter (34). They analyzed the radial breathing mode of the CNTs as they were filled with water, and they measured water transport at various temperatures. The results show both an exquisite sensitivity to diameter and substantially larger temperature elevations of the melting transition than theoretically predicted. Reversible melting was observed at 138 and $102{ }^{\circ} \mathrm{C}$ for water in 1.05 and $10.6 \mathrm{~nm}$ CNTs, respectively. A near-ambient phase change at $15^{\circ} \mathrm{C}$ was observed in $1.52 \mathrm{~nm}$ CNTs, whereas inside $1.24 \mathrm{~nm}$ CNTs freezing was suppressed below $-30{ }^{\circ} \mathrm{C}$. This is interesting from a fundamental point of view, because fluid phase transitions inside single, isolated CNTs are predicted to deviate substantially from bulk ones (93), and also from a practical point of view, because CNT-based membranes would cease to function if the water confined within the individual pores was to solidify.

The availability of single-pore platforms could allow the generalization of the proofof-concept results reported by Majumder et al. (94), who took advantage of the possibility of functionalizing the ends of the CNTs embedded in a membrane to control, via applied voltages, the transport of ruthenium bipyridine hexahydrate. These data suggest that the transport selectivity of advanced nano-structured membranes can be achieved by taking advantage of functionalization. Experimental data are available for a single-pore drilled in graphene because of the potential application of such devices in DNA sequencing applications $(26,28)$. For example, Garaj et al. (51) used chemical vapor deposition $(95,96)$ to produce $0.5 \cdot 0.5 \mathrm{~mm}^{2}$ graphene membranes, mounted on a free-standing $\mathrm{SiN}_{\mathrm{x}}$ layer. They found a linear relationship between the ionic conductivity across the membranes with one single pore, created using electron beam drilling, and the pore diameter. These results suggest that the effective insulating thickness of the graphene membrane is $\sim 0.6$ $\mathrm{nm}$, consistent with atomistic MD simulations (97), which indicate that the distance between water and carbon atoms on both sides of a layer of graphene is $\sim 0.3 \mathrm{~nm}$ (see Figure 2). The linear relationship between ionic conductivity and pore diameter, rather than pore area, is expected for atomically short nanopores in which the resistance is dominated by the 'access resistance' (98), and it is consistent with 
the solution of the Laplace equation across idealized uncharged insulating membranes. The experimental data were obtained for $\sim 5 \mathrm{~nm}$ diameter pores (51), and more recently as little as $\sim 3 \mathrm{~nm}(52)$. While these results are impressive, for the development of RO membranes it would be beneficial to push the limits of these experiments to sub-nanometer pores, with a high degree of control also on the chemical functionalization of the pore edges. Such data would be directly comparable to simulation results.

While most of the research summarized above aims to improve RO membranes, it should also be pointed out that additional reductions in the pressure drop across a membrane might not yield the expected improvement in practice. Thermodynamics is the culprit, as the difference in osmotic pressure between purified and salty water will always cause a significant pressure drop across the membrane. Several researchers estimated the benefits, in terms of overall energy consumption, which can be expected from new membranes. Considering that in practical water desalination operations the salt rejection across a RO membrane is $\sim 99 \%$ or higher (99), the minimum applied hydraulic pressure required to generate desalinated water from a brine solution will have to be larger than the osmotic pressure difference between permeate and retentate solutions. The pressure drop across the membrane will be added to this minimum. The state of the art in RO has reached energy efficiencies that are not considered too far from the thermodynamic limit of a single-stage operation (in a single stage operation, salty water is fed to the separation, the retentate is the more concentrated solution to be disposed of, and the permeate is the desalinated water) (100). Scientists wonder what more can be achieved with expensive nano-structured membranes? Perhaps better energy efficiency can be achieved by changing the configuration of the desalination processes. For example, Lin and Elimelech (101) recently compared the energy requirements in multi-stage processes, either in direct pass or in closed circuit realizations, and quantified their benefits compared to single stage processes. Others remain convinced that ' $R O$ membranes have tremendous room for improvement', and suggest that new nano-structured membranes resistant to chemical degradation can be obtained using graphene (7). Cohen-Tanugi and Grossman modeled the performance of a RO plant taking into consideration feed salinity and flow rate, concentration polarization across the membrane, membrane permeability and a few other key parameters, although they omitted the effect of fouling. Their calculations suggested that a membrane 3 times more permeable than existing ones could reduce the pressure required for seawater treatment by $15 \%$. However, they also pointed out that new membranes 1000 times more permeable than the current ones would not lead to great improvements in energy efficiency (102).

Regarding fouling, Belfort and his group recently proposed an experimental combinatorial method for synthesizing, screening, and discovering antifouling surfaces $(103,104)$. The group developed a high-throughput platform that employs atmospheric-pressure plasma polymerization to modify commercial poly(ether sulfone) (PES) membranes. They investigated the effect of carbon chain length of the 
functional groups and they found that a minimal chain length of 4-6 carbons is required for hydroxyl and poly(ethylene glycol) monomers to achieve anti-fouling. When the chains are shorter or longer, the performance decreases (104). More recently, they quantified the effect of new functional graft-polymerized groups. They quantified performance in terms of fouling index, solute selectivity, and flux (104). To rationalize the results, they calculated the Hansen solubility parameter (105) of the synthesized monomers and found that the best-performing membranes had functional groups whose Hansen solubility parameter was closest to that of water. While this pragmatic observation is useful for securing progress, it suggests that local properties, in particular water-monomer interactions, are responsible for rather large-scale phenomena such as protein adsorption (106). Many studies have been conducted on water properties at the interface with solid substrates (107, 108), and perhaps the most intriguing breakthroughs from recent discoveries is the correlation between water density fluctuations near an interface and the ability of various macromolecules to adsorb (109), and perhaps even the solubility of gases in confined water (110). The latter study suggests that the proximity of two surfaces not only affects the structure and dynamics of confined water, but also the ability of water molecules to fluctuate. It would be interesting to determine how the proximity of functional groups affect water properties at an interface, and how these effects are related to data such as those presented by Belfort and coworkers.

\section{Alternative water desalination processes: capacitive deionization}

An alternative approach to desalinate water is capacitive deionization, CD (111, 112). The process came back to the spotlight recently, with the enhanced interest in electric double layer capacitors, EDLCs, for energy storage (113). CD and EDLCs both work via the accumulation of ions at charged electrode-electrolyte interfaces (114). CD can be attractive compared to RO because its goal is to collect ions from salty water, rather than process all water molecules through membranes. Because the amount of ions that can be captured using CD scales with the electrode's surface area, conductive porous materials such as active carbons with high surface area are used $(115,116)$. Challenges to bring $\mathrm{CD}$ to fruition include the maximization of the capacitance, to capture more ions, and synchronization of water flow through the device (117). In fact, once ions are adsorbed on the electrodes, the latter need to be regenerated (118), making $C D$ processes discontinuous, unless complicated operations are designed (e.g., desalination with wires (119)).

Recently the community has made significant strides that can benefit CD. In particular, the energy barriers encountered by different ions as they enter narrow carbon-based pores from bulk solutions were quantified (120-122), as well as ionion and ion-water correlations under confinement (123-125). Kalluri et al. (126) reported a combination of electrochemical experiments, supported by atomistic simulations, to quantify the effects of pore size and surface charge density on the capacitance of graphitic nanoporous carbon electrodes. Carbon foams were produced with two distinct pore size distributions (non-activated materials characterized by sub-nm pores and activated ones with pores as wide as $\sim 2 \mathrm{~nm}$ ). 
The cyclic voltammetry data obtained for the activated pores showed ideal (linear) capacitive behavior (i.e., rectangular shape and charging/discharging currents independent on the applied potential window), while the correspondent data obtained for the non-activated pores showed strong increases in capacitance $(>1$ order of magnitude) when wide potential sweeps were applied. The latter results depend on the ions present in solution, with the peak that marked the transition from low to high capacitance shifting to more negative potentials as the strength of the hydration shell increased (no obvious correlation was found with the size of the ions or the size of the hydrated ions). These data were consistent with simulation results for the free energy barriers experienced by ions entering negatively charged CNTs (121), as well as with simulations reporting the density of ions confined within carbon slit-shaped pores at different surface charge densities $(123,127)$.

Ho and Striolo (128), using equilibrium MD simulations, found that the structure and orientation of interfacial water, as well as the accumulation of $\mathrm{NaCl}$ ions near the interface, depend on electrode patterning (the electrodes were graphene layers, patterned by removing selected carbon atoms). These effects led to significant variations in the capacitance of the electrodes. The Authors also recently proposed, in another computational effort, to combine some features of membrane desalination and some of $\mathrm{CD}$ to obtain a continuous desalination device (129). In the composite cell proposed (see Figure 3), the charged electrodes extract the ions from the feed-water; the non-charged graphitic membranes manipulate the electric double layer and prevent the ions, once captured, from returning to the central pore; the resultant short distance between captured ions and charged electrodes increases the capacitance; water slips at the walls of the slit-shaped channels (24), which allows for continuum operation. Based on these simulations, the cell can be operated continuously, could achieve $100 \%$ charge efficiency and recover $70 \%$ of the water initially loaded to the system with $100 \%$ salt rejection at salinity less than $10 \mathrm{~g} / \mathrm{L}$, and is expected to yield $30 \mathrm{~L}$ of desalinated water per $\mathrm{cm}^{2}$ of membrane, per day, per MPa of pressure drop (129). Of course, experimental investigations are needed to bring to practical fruition this device, and to provide more realistic quantifications of the promising indicators just summarized. 


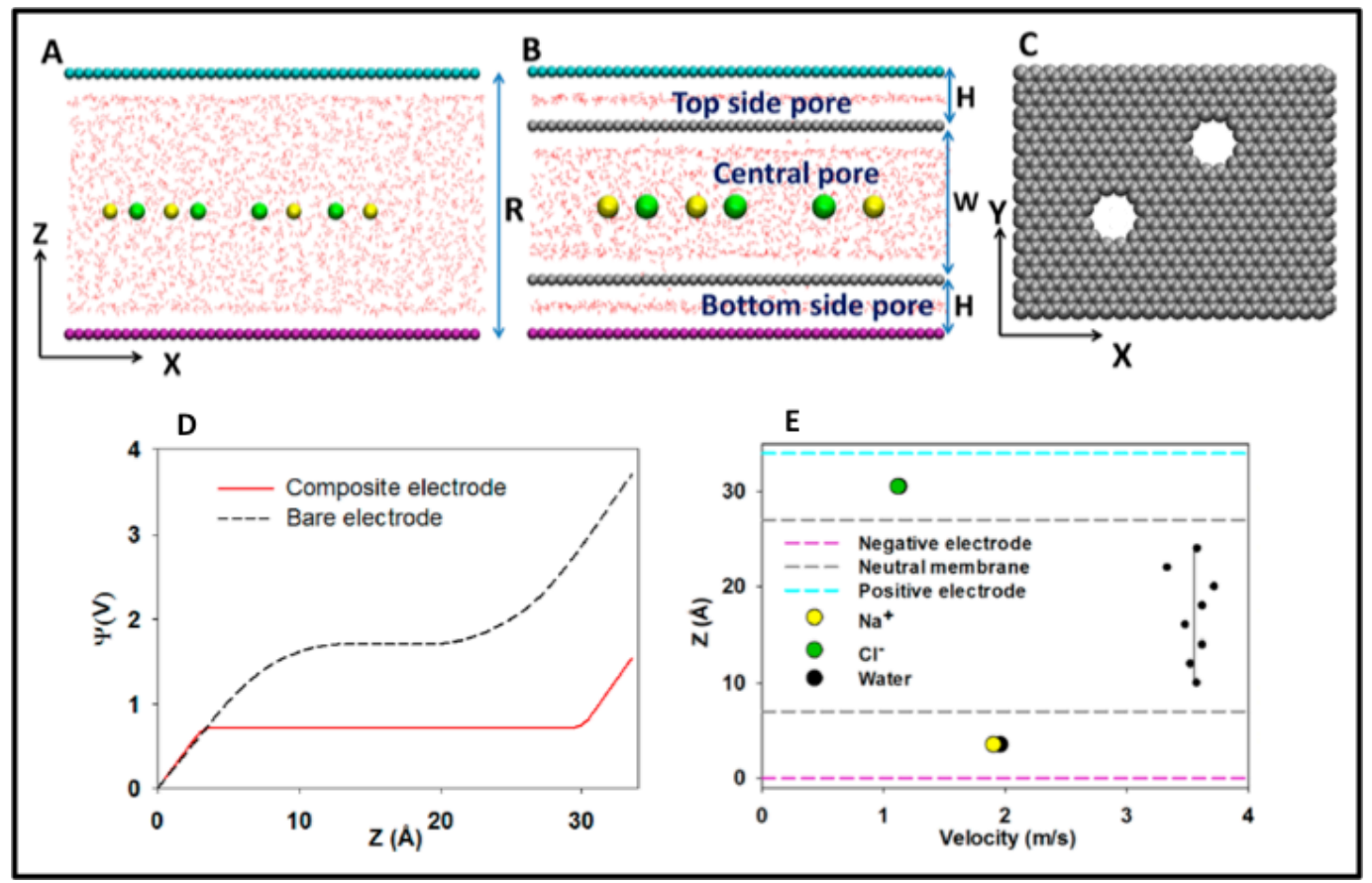

Figure 3. Top panels: schematic of the simulated water desalination cell. Panels A and B provide side views of two cells: the bare one, in which the positive and negative electrodes (cyan and purple, respectively) face across the pore volume, and the composite one, in which graphene membranes

(panel C) are used to separate the central pore from top and bottom side pores. In panel D the electrical potential profiles are calculated along the direction perpendicular to the electrode surface

for bare and composite electrodes. In panel E the steady-state velocities calculated for water molecules and ions within the composite electrode are reported as a function of the position within the composite cell. Reproduced from Ref. (129), figures 1, 4, and 5.

\section{Frontiers of simulation studies}

Although atomistic simulations have contributed to many areas of scientific endeavor such as the development of solar cells (130), batteries (131), and new catalysts $(132,133)$ to name just a few, one needs to recognize that computational approaches have a number of limitations. Four key restrictions relate to: (a) the accuracy of the potential energy surfaces; (b) the algorithms and sampling techniques used; (c) the description of the material, and (d) the finiteness of computational resources. We now briefly discuss how such limitations can be overcome to benefit water desalination: 


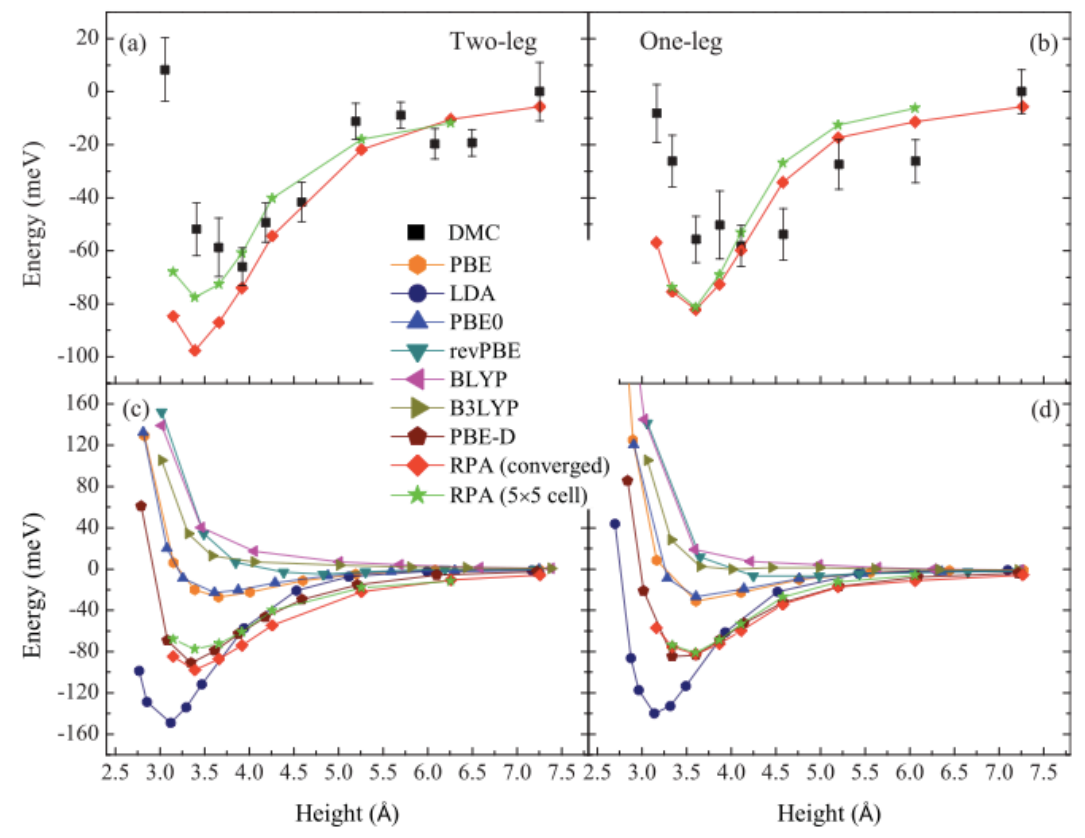

Figure 4. Adsorption energy versus 0 atom height for a single water on graphene obtained with various ab initio electronic structure based methods. (a) and (c) are for a so-called "two-leg" configuration in which both hydrogens of the water molecule are directed at the surface. (b) and (d) are for a so-called "one-leg" configuration in which only one $\mathrm{OH}$ bond is directed at the surface. In the upper panels the accurate reference methods of diffusion quantum Monte Carlo (DMC) and the random phase approximation (RPA) are compared. In the lower panels, RPA and various forms of density functional theory (i.e., different xc functionals) are compared. Reproduced from Ref. (134).

a) Accuracy of the potential energy surfaces: In all simulation studies one must ensure that the theoretical approach used can faithfully describe the systems being considered. Most simulations discussed so far have been performed with empirical potentials (i.e., classical force fields), which are sensitive to the choice of the potentials used. Many classical models are available for the description of waterwater interactions $(135,136)$, and some have been used to investigate interfacial systems (107).

Of the many studies, Ho and Striolo compared the predictions obtained by equilibrium MD simulations for water at contact with graphene (137). Among the models considered, it was found that the SPC/E, TIP4P/2005, SPC/Fw, TIP4P/2005f, and SWM4_DP models yielded structures of interfacial water on neutral, negatively and positively charged graphene that were very similar to each other. Somewhat different predictions, in particular for the dynamics of interfacial water, were obtained when the TIP5P and the TIP3P models of water were considered. Liu and Patay compared how different water models performed to predict water transport through CNTs (138). The fastest model (TIP3P) had a flow rate approximately five times greater than the slowest (TIP4P/2005), which was related to a different structure in confinement. Often, it is suggested that polarization effects, important to describe the water-vacuum interface (139), could be essential to reliably describe the graphene-water interface. Ho and Striolo 
attempted to quantify effects due to water polarizability on both structure and dynamics of water near graphene, neutral and charged (140). The results suggested that polarizability might not be important at the water-graphene interface. However, to judge which models are best at reproducing realistic scenarios, very detailed experimental information is required. Lacking this information, detailed ab initio simulations could yield important insights.

Besides water-water interactions, it could also be important to describe correctly carbon-carbon interactions. Once again there are many carbon potentials of varying levels of sophistication to choose from. We will not discuss the details of the carbon potentials here except to mention two very recent force field studies for water in CNTs and on graphene, which have shown that the motion of the water can couple to the phonon modes of the graphene sheet or carbon nanotube $(141,142)$. This suggests that an accurate description of the phonon spectrum of the carbon material is a prerequisite for faithful predictions of water transport properties.

Once the models for water and carbon are chosen, one has to consider the nature of the water-carbon interaction. Unfortunately this is still not very well quantified, as adsorption energies of isolated water molecules on well-defined surfaces are not well established (143). In the absence of better alternatives, the water-carbon interaction used in most studies was obtained by fitting to the macroscopic contact angle of water droplets (97). This approach has proved useful. However, given that the contact angle arises from a balance of surface energies and because different water and carbon models yield different surface energies, the water-carbon interaction depends on the models used to describe water and carbon. This is obviously not a very satisfactory situation, and more troublesome is that the experimental contact angle for water droplets depends sensitively on experimental conditions (particularly impurities and defects) and is itself a matter of debate (144146). Alternatively, the water-carbon interaction can be obtained directly from the electron density using, for example, Kohn-Sham density functional theory (DFT) without relying on empirical parameters. DFT has been widely used to study water on carbonaceous surfaces $(134,147-153)$.

However, practical DFT calculations do contain approximations, most notably in how electron exchange and correlation effects are treated within the so-called exchange-correlation (xc) functional. Nowadays there is an almost endless list of xc functionals $(154,155)$, and especially for weak interaction systems such as water adsorption on carbonaceous surfaces the results can vary significantly from one functional to the next. In Figure 4 we report, as an illustration, the predicted adsorption energy of a single water monomer on graphene as obtained from a variety of DFT xc functionals (as well as other ab initio methods). It can be seen that the predicted adsorption energies vary from $\sim 150 \mathrm{meV}(\sim 4 \mathrm{kcal} / \mathrm{mol})$ right down to nothing at all! Of course the xc functionals that predict no binding are not appropriate for this system. The lack of any binding arises because these functionals do not account for van der Waals forces, an issue that DFT developers are addressing with vigor (156). Nonetheless it is clear that at present one cannot easily 
turn to DFT for a benchmark of the water-carbon interaction. With this in mind there have been a number of other studies using yet more sophisticated electronic structure theories aimed at establishing an accurate theoretical reference for the interaction of water with sp2-bonded carbon $(134,157-162)$. This has involved a very broad range of approaches, such as periodic quantum Monte Carlo, random phase approximation calculations, coupled cluster, and symmetry adapted perturbation theory on acene clusters. $\mathrm{Wu}$, Aluru and co-workers tabulated many of the results (157), and although a consensus has yet to be reached, these high-level calculations yield values in the $70-120 \mathrm{meV}$ range $(\sim 2-4 \mathrm{kcal} / \mathrm{mol})$.

In desalination studies, it is necessary to also describe ion-water and ion-ion interactions. With regard to this issue it is important to point out that the community has recently realized that widely used classical force fields might not be sufficiently accurate to describe the solubility of salt in water, because they were parameterized to reproduce properties of individual salts $(163,164)$. Ding et al. compared the predictions of force field and ab initio MD for the self-diffusion of water in the presence of $\mathrm{NaCl}$ and CsI salts (165). They suggested that only ab initio simulations can reproduce the qualitative trends observed experimentally (166). They then showed that the anomalous water diffusion in salt solutions has a subtle origin, involving electronic properties of water. Classical force fields cannot easily describe such effects, which can, of course, be captured by ab initio methods. While this might not be a significant limitation when single ions are considered, they could compromise the reliability of the simulation predictions when one is interested in simulating the accumulation of ions within a charged carbon-based pore (of relevance in $\mathrm{CD}$ ) and when one is interested in quantifying concentration effects (i.e., screening) on ions rejection. We refer the interested reader to a recent contribution by Jiang et al. (167), who found that for aqueous systems containing $\mathrm{NaCl}$ the polarizable force field of Kiss and Baranyai (168) yields much better predictions for quantities such as salt solubility, solution density and viscosity as a function of system temperature, pressure, and salt concentration, than fixed point nonpolarizable models.

DFT approaches have been employed to better understand ion-water interactions, and in particular the hydration shell. For a brief review of DFT studies of aqueous solutions the interested reader is referred to ref. (169). Of course the limits of DFT for the description of pure water, related to the use of an approximate xc functional, apply also to salt solutions. Bankura et al. recently showed that hybrid functionals (i.e., with the addition of a fraction of exact exchange) that also account for for Van der Waals forces can provide excellent agreement with experiments both for the structure and electronic properties of a chloride ion solvated in water (170). Although hybrid functionals are very demanding (computing times are at least an order of magnitude larger than standard semi-local xc functionals), these results are very encouraging concerning the accurate description of both structure and dynamics of aqueous salt solutions. For instance, Li et al. recently used AIMD to explore the mechanisms of selective ion transport in hydrophobic nanochannels, including CNTs (using a standard semi-local functional) (171). They showed that 
CNTs of suitable size provide very selective ion channels. Such predictions depend strongly on the accuracy of the description of the hydrated ion structure and dynamics, for which AIMD generally proves to be accurate. To illustrate the importance of appropriately describing carbon-water interactions, we refer to a recent manuscript, which discussed the possible reasons why the presence of cations could block water flow through narrow CNTs (172). The Authors used DFT to improve the force fields employed in classical MD simulations. Their results suggest that specific attractions can arise between cations and the aromatic rings located at the opening of $(6,6)$ single walled CNTs, which can block the pores to water. Perhaps these effects could explain why water permeability through CNT membranes was reported to decay after some time $(29,30)$.

Finally, we note that although DFT simulations of water/carbon interfaces are not problem-free, they can a priori account for water dissociation and polarizability effects. Because both of these issues represent major challenges for traditional force field approaches, with the improvements in the DFT description of van der Waals forces and with the great advances in computing power and algorithmic efficiencies, it seems inevitable that DFT will become increasingly useful in tackling wet carbonaceous interfaces.

b) Algorithms and sampling techniques: Typically in a simulation of a solid-liquid interface thermostats will be applied to control the temperature of the system. Considerable research goes into thermostat development (see e.g. (173)) but generally they can alter the dynamics of the system, to some extent, and so this important technical issue deserves some comment. Thomas and Corry explicitly considered the effect of various algorithms, commonly used to control the temperature in MD simulations, on the predicted water flux across CNT membranes (174). They considered two groups of algorithms. In the first group the molecular velocities are rescaled at regular intervals to maintain the effective system temperature within the desired target (e.g., the Berendsen thermostat). In the second group the molecular velocities are randomized rather than scaled (e.g., the Andersen thermostat). The results showed that the fluxes of water molecules across individual $(10,10)$ CNTs for a given pressure drop significantly depend on the thermostat (direct rescaling yields a flux three times as large as that obtained using a Langevin thermostat, all other system parameters being equal). The Authors also investigated the permeability as a function of the membrane thickness, and found that the results do not show much reduced permeability when velocity rescaling algorithms are applied, while randomized velocities algorithms lead to decreases in permeability with membrane thickness. As discussed in section 2, water flow through nanometric CNTs is limited by entrance viscous dissipation $(31,35)$ and should not depend on tube length for the short tubes considered in the study by Thomas and Corry (38). Therefore only the velocity rescaling algorithms reproduce the expected behavior. However this does not mean that randomized velocities algorithms are fundamentally flawed: for instance, Joly studied capillary filling of CNTs with water and obtained consistent results when he compared two different thermostating algorithms: Nosé-Hoover applied only to the degrees of freedom 
perpendicular to the flow and a Dissipative Particle Dynamics (DPD) thermostat (35). The DPD thermostat is very similar to the Langevin thermostat, but it preserves hydrodynamics by construction, as the friction force is proportional to the velocity difference between neighbor atoms, so that it could provide an interesting way to thermostat liquids in complex, 3D flows. Overall, these observations should be taken into consideration when insights from simulations are used to interpret experiments. It should also be remembered that it is not clear which algorithms yield the most realistic results, as a direct comparison against experimental data on single CNTs is not yet possible.

Regarding the choice of algorithms to investigate molecular phenomena related to $\mathrm{CD}$ applications, it is common practice to apply a uniform charge density on graphitic materials to replicate an applied potential $(126,128,129)$. However, this might not always be a realistic representation of the processes. Merlet et al. (175, 176) showed that it is possible to conduct simulations by imposing a constant potential across the simulation cell instead. The distribution of ions within the cell depended on the algorithm applied, especially at high surface charge density. While the algorithm is not expected to affect the results at moderate surface charge densities such as $0.26 \mathrm{e} / \mathrm{nm}^{2}$, one should be careful in securing reliable connections with experiments when high potentials are considered.

c) Description of the material: Computer simulations inevitably involve simplified and very often idealized models of the system under study. Therefore simulation studies are best suited to better understanding the mechanisms responsible for and underlying physical principles of experimental observations. In such cases, one does not need to replicate the features of an entire RO membrane or CD device. However, it is becoming increasingly important to be able to directly apply the simulations to experiments while reproducing as many features as possible. This is key, e.g., to help settle arguments such as the large variations reported in water fluxes through CNTbased RO membranes (discussed in section 2). Gubbins and coworkers developed advanced algorithms for reproducing the sub nm-level features of carbon adsorbents $(177,178)$. Similar approaches should be attempted to replicate the physical properties of GO stacks used in RO membranes, to better understand how processing conditions determine the performance of such devices. Promising results have been obtained when similar approaches were applied to describe the structural evolution of the electrodes used in EDLCs (179), for example. For the applications discussed in this review, it will also be necessary to include a detailed description of the type, density and distribution of defects, including oxygenated sites and vacancies, that are found in the carbon-based substrates, as these are likely to affect the performance of both RO membranes and CD devices. Once a realistic description of the materials used for water-desalination is available, multi-scale models, not discussed within this review, will perhaps become useful for discovering new water desalination processes.

d) Finiteness of computational resources: Although computing power continues to rapidly increase with the development of new computing architectures, it is likely 
that predictions from simulations will always come with uncertainties. With this in mind it is highly desirable to develop procedures that quantify the propagation of uncertainty from force fields to predicted properties. Angelikopoulos et al. proposed one such approach, based on the Bayesian framework (180). The Authors developed a high-performance-computing algorithm for propagating the uncertainties. The approach can be used to estimate simulation model parameters from available experimental data, and also to compare the relative uncertainties of computational predictions, due to both force fields and algorithms, and experimental observables. The development of machine learning potentials, which come with explicit error estimates also looks like a very promising and powerful avenue for research in this area, especially given the relentless increases in computational power and data storage capacity we are witnessing $(181,182)$.

\section{Conclusions}

We have reviewed some recent experiments and computational contributions related to the water-energy nexus, and in particular water desalination. Emphasis was given to membranes for reverse osmosis, and devices for capacitive desalination. These technologies are leading to the development of increasingly structured nanoporous carbon-based materials. Therefore understanding at the fundamental level how water and ions behave in contact with carbon substrates, and how external fields, the presence of defects such as oxygenated sites, impurities, etc., affect this behavior is critical. While considerable insights can be achieved from qualitative comparison between experimental observations and simulations, to truly impact the field we believe that more robust connections between experimental devices and computational approaches are needed. We have discussed some possible ways in which this can be achieved, including experimental observations for single-pore systems and more reliable force fields built with insights from DFT, as well as DFT and beyond DFT approaches. Although ab initio techniques are extremely versatile, empirical approaches remain useful for studying large systems. While we did not discuss multi-scale approaches here, we anticipate that they will become critical for better understanding devices such as graphene oxide stacks used in reverse osmosis.

Open questions that remain to be addressed include: Why does the water flow through CNT-based membranes quickly stop? If clogging is the culprit, how can we design processes to clean a $2 \mathrm{~nm}$ pore? How can simulations provide insights to improve the mechanical strength and the resistance to degradation of membranes for water desalination? And, still even what is the true interaction strength between water and carbonaceous surfaces? Surely, a synergistic approach that combines multi-scale simulations and experiments can help to address these questions as well. It is also highly probable that new 2D materials beyond carbon will become useful for the water-energy nexus (183). In particular, much emphasis is currently being given to understanding and quantifying the material properties of "white graphene' (hexagonal boron nitride) and transition metal dichalcogenide 
monolayers. Perhaps the insights gleaned from carbon-based materials and devices can be helpful in understanding and screening these and other materials.

\section{Acknowledgements}

A.S. acknowledges financial support generously provided by the US Department of Energy, Marie Curie Career Integration Grant, the European Union Horizon 2020 Research and Innovation Programme under research grant 640979, the UK Engineering and Physical Sciences Research Council, and Halliburton. A.S. is grateful to his group members for their contribution to the research summarized here, in particular, Dr. Tuan A. Ho, Dr. Dimitrios Argyris, Dr. N.R. Tummala, and Ms. Anh Phan.

A.M. is supported by the European Research Council under the European Union's Seventh Framework Programme (FP/2007-2013) / ERC Grant Agreement number 616121 (HeteroIce project). A.M is also supported by the Royal Society through a Royal Society Wolfson Research Merit Award.

L.J. is supported by the French Ministry of Defense through the project DGA ERE number 2013.60.0013, and by the LABEX iMUST (ANR-10-LABX-0064) of Université de Lyon, within the program "Investissements d'Avenir" (ANR-11-IDEX-0007) operated by the French National Research Agency (ANR).

\section{References}

1. Stover RL. 2007. Seawater reverse osmosis with isobaric energy recovery devices. Desalination. 203:168-75.

2. Ghaffour N, Missimer TM, Amy GL. 2013. Technical review and evaluation of the economics of water desalination: Current and future challenges for better water supply sustainability. Desalination. 309:197-207.

3. Fritzmann C, Lowenberg J, Wintgens T, Melin T. 2007. State-of-the-art of reverse osmosis desalination. Desalination. 216:1-76.

4. Semiat R. 2008. Energy Issues in Desalination Processes. Environ Sci Technol. 42:8193-201.

5. Elimelech M, Phillip WA. 2011. The Future of Seawater Desalination: Energy, Technology, and the Environment. Science. 333:712-17.

6. Lee KP, Arnot TC, Mattia D. 2011. A review of reverse osmosis membrane materials for desalination-Development to date and future potential. J Membrane Sci. 370:1-22.

7. Cohen-Tanugi D, Grossman JC. 2015. Nanoporous graphene as a reverse osmosis membrane: Recent insights from theory and simulation. Desalination. 366:59-70.

8. Subramani A, Jacangelo JG. 2015. Emerging desalination technologies for water treatment: A critical review. Water Res. 75:164-87.

9. Daer S, Kharraz J, Giwa A, Hasan SW. 2015. Recent applications of nanomaterials in water desalination: A critical review and future opportunities. Desalination. 367:37-48. 
10. Das R, Ali ME, Abd Hamid SB, Ramakrishna S, Chowdhury ZZ. 2014. Carbon nanotube membranes for water purification: A bright future in water desalination. Desalination. 336:97-109.

11. Rodríguez-Calvo A, Silva-Castro GA, Osorio F, González-López J, Calvo C. 2014. Novel Membrane Materials for Reverse Osmosis Desalination. Hydrol Current Res. 5:1-7.

12. Muller EA. 2013. Purification of water through nanoporous carbon membranes: a molecular simulation viewpoint. Curr Opin Chem Eng. 2:223-28.

13. Guo S, Eric R. Meshot, Tevye Kuykendall, Stefano Cabrini, Fornasiero F. 2015. Nanofluidic Transport through Isolated Carbon Nanotube Channels: Advances, Controversies, and Challenges. Advanced Materials. 27:5726-37.

14. Kannam SK, Todd BD, Hansen JS, Daivis PJ. 2013. How fast does water flow in carbon nanotubes? J Chem Phys. 138:094701.

15. Park HG, Jung Y. 2014. Carbon nanofluidics of rapid water transport for energy applications. Chem Soc Rev. 43:565-76.

16. Thomas M, Corry B, Hilder TA. 2014. What Have We Learnt About the Mechanisms of Rapid Water Transport, Ion Rejection and Selectivity in Nanopores from Molecular Simulation? Small. 10:1453-65.

17. Ebro H, Kim YM, Kim JH. 2013. Molecular dynamics simulations in membrane-based water treatment processes: A systematic overview. J Membrane Sci. 438:112-25.

18. Bocquet L, Charlaix E. 2010. Nanofluidics, from bulk to interfaces. Chem Soc Rev. 39:1073-95.

19. Pascal TA, Goddard WA, Jung Y. 2011. Entropy and the driving force for the filling of carbon nanotubes with water. P Natl Acad Sci USA. 108:11794-98.

20. Hummer G, Rasaiah JC, Noworyta JP. 2001. Water conduction through the hydrophobic channel of a carbon nanotube. Nature. 414:188-90.

21. Kalra A, Garde S, Hummer G. 2003. Osmotic water transport through carbon nanotube membranes. P Natl Acad Sci USA. 100:10175-80.

22. Alexiadis A, Kassinos S. 2008. Molecular Simulation of Water in Carbon Nanotubes. Chem Rev. 108:5014-34.

23. Striolo A. 2006. The mechanism of water diffusion in narrow carbon nanotubes. Nano Lett. 6:633-39.

24. Falk K, Sedlmeier F, Joly L, Netz RR, Bocquet L. 2010. Molecular Origin of Fast Water Transport in Carbon Nanotube Membranes: Superlubricity versus Curvature Dependent Friction. Nano Lett. 10:4067-73.

25. Falk K, Sedlmeier F, Joly L, Netz RR, Bocquet L. 2012. Ultralow Liquid/Solid Friction in Carbon Nanotubes: Comprehensive Theory for Alcohols, Alkanes, OMCTS, and Water. Langmuir. 28:14261-72.

26. Joseph S, Aluru NR. 2008. Why are carbon nanotubes fast transporters of water? Nano Lett. 8:452-58.

27. Striolo A. 2007. Water self-diffusion through narrow oxygenated carbon nanotubes. Nanotechnology. 18:475704.

28. He ZJ, Corry B, Lu XH, Zhou J. 2014. A mechanical nanogate based on a carbon nanotube for reversible control of ion conduction. Nanoscale. 6:3686-94. 
29. Majumder M, Chopra N, Andrews R, Hinds BJ. 2005. Nanoscale hydrodynamics: Enhanced flow in carbon nanotubes (vol 438, pg 44, 2005). Nature. 438:930-30.

30. Majumder M, Chopra N, Andrews R, Hinds BJ. 2005. Nanoscale hydrodynamics - Enhanced flow in carbon nanotubes. Nature. 438:44-44.

31. Sisan TB, Lichter S. 2011. The end of nanochannels. Microfluid Nanofluid. 11:787-91.

32. Holt JK, Park HG, Wang YM, Stadermann M, Artyukhin AB, Grigoropoulos CP, et al. 2006. Fast mass transport through sub-2-nanometer carbon nanotubes. Science. 312:1034-37.

33. Qin XC, Yuan QZ, Zhao YP, Xie SB, Liu ZF. 2011. Measurement of the Rate of Water Translocation through Carbon Nanotubes. Nano Lett. 11:2173-77.

34. Agrawal KV, Shimizu S, Drahushuk LW, Kilcoyne D, Strano MS. 2015. Observation of extreme phase transition temperatures of water confined inside isolated carbon nanotube nanopores. submitted.

35. Joly L. 2011. Capillary filling with giant liquid/solid slip: Dynamics of water uptake by carbon nanotubes. J Chem Phys. 135:214705.

36. Gravelle S, Joly L, Detcheverry F, Ybert C, Cottin-Bizonne C, Bocquet L. 2013. Optimizing water permeability through the hourglass shape of aquaporins. P Natl Acad Sci USA. 110:16367-72.

37. Gravelle S, Joly L, Ybert C, Bocquet L. 2014. Large permeabilities of hourglass nanopores: From hydrodynamics to single file transport. J Chem Phys. 141:18C526.

38. Walther JH, Ritos K, Cruz-Chu ER, Megaridis CM, Koumoutsakos P. 2013. Barriers to Superfast Water Transport in Carbon Nanotube Membranes. Nano Lett. 13:1910-14.

39. Thomas JA, McGaughey AJH. 2009. Water Flow in Carbon Nanotubes: Transition to Subcontinuum Transport. Phys Rev Lett. 102:184502.

40. Majumder M, Chopra N, Hinds BJ. 2011. Mass Transport through Carbon Nanotube Membranes in Three Different Regimes: Ionic Diffusion and Gas and Liquid Flow. Acs Nano. 5:3867-77.

41. Thomas M, Jayatilaka D, Corry B. 2013. How does overcoordination create ion selectivity? Biophys Chem. 172:37-42.

42. Sharma S, Debenedetti PG. 2012. Evaporation rate of water in hydrophobic confinement. P Natl Acad Sci USA. 109:4365-70.

43. Suk ME, Aluru NR. 2013. Molecular and continuum hydrodynamics in graphene nanopores. Rsc Adv. 3:9365-72.

44. Suk ME, Aluru NR. 2014. Ion transport in sub-5-nm graphene nanopores. J Chem Phys. 140:084707.

45. Cohen-Tanugi D, Grossman JC. 2012. Water Desalination across Nanoporous Graphene. Nano Lett. 12:3602-08.

46. O'Hern SC, Jang D, Bose S, Idrobo JC, Song Y, Laoui T, et al. 2015. Nanofiltration across Defect-Sealed Nanoporous Monolayer Graphene. Nano Lett. 15:3254-60.

47. Konatham D, Yu J, Ho TA, Striolo A. 2013. Simulation Insights for GrapheneBased Water Desalination Membranes. Langmuir. 29:11884-97. 
48. O'Hern SC, Boutilier MSH, Idrobo JC, Song Y, Kong J, Laoui T, et al. 2014. Selective Ionic Transport through Tunable Subnanometer Pores in Single-Layer Graphene Membranes. Nano Lett. 14:1234-41.

49. Pendergast MM, Hoek EMV. 2011. A review of water treatment membrane nanotechnologies. Energ Environ Sci. 4:1946-71.

50. Koenig SP, Wang LD, Pellegrino J, Bunch JS. 2012. Selective molecular sieving through porous graphene. Nat Nanotechnol. 7:728-32.

51. Garaj S, Hubbard W, Reina A, Kong J, Branton D, Golovchenko JA. 2010. Graphene as a subnanometre trans-electrode membrane. Nature. 467:190-U73.

52. Garaj S, Liu S, Golovchenko JA, Branton D. 2013. Molecule-hugging graphene nanopores. P Natl Acad Sci USA. 110:12192-96.

53. Merchant CA, Healy K, Wanunu M, Ray V, Peterman N, Bartel J, et al. 2010. DNA Translocation through Graphene Nanopores. Nano Lett. 10:2915-21.

54. Nair RR, Wu HA, Jayaram PN, Grigorieva IV, Geim AK. 2012. Unimpeded Permeation of Water Through Helium-Leak-Tight Graphene-Based Membranes. Science. 335:442-44.

55. Dikin DA, Stankovich S, Zimney EJ, Piner RD, Dommett GHB, Evmenenko G, et al. 2007. Preparation and characterization of graphene oxide paper. Nature. 448:457-60.

56. Eda G, Chhowalla M. 2010. Chemically Derived Graphene Oxide: Towards Large-Area Thin-Film Electronics and Optoelectronics. Advanced Materials. 22:2392-415.

57. Kim HW, Yoon HW, Yoon SM, Yoo BM, Ahn BK, Cho YH, et al. 2013. Selective Gas Transport Through Few-Layered Graphene and Graphene Oxide Membranes. Science. 342:91-95.

58. Park HB. 2014. Graphene-based membranes - a new opportunity for CO2 separation. Carbon Manag. 5:251-53.

59. Li H, Song ZN, Zhang XJ, Huang Y, Li SG, Mao YT, et al. 2013. Ultrathin, Molecular-Sieving Graphene Oxide Membranes for Selective Hydrogen Separation. Science. 342:95-98.

60. Han Y, Xu Z, Gao C. 2013. Ultrathin Graphene Nanofiltration Membrane for Water Purification. Adv Funct Mater. 23:3693-700.

61. Sun PZ, Zhu M, Wang KL, Zhong ML, Wei JQ, Wu DH, et al. 2013. Selective Ion Penetration of Graphene Oxide Membranes. Acs Nano. 7:428-37.

62. $\mathrm{Hu} \mathrm{M}, \mathrm{Mi}$ BX. 2013. Enabling Graphene Oxide Nanosheets as Water Separation Membranes. Environ Sci Technol. 47:3715-23.

63. Huang HB, Mao YY, Ying YL, Liu Y, Sun LW, Peng XS. 2013. Salt concentration, $\mathrm{pH}$ and pressure controlled separation of small molecules through lamellar graphene oxide membranes. Chem Commun. 49:5963-65.

64. Joshi RK, Carbone P, Wang FC, Kravets VG, Su Y, Grigorieva IV, et al. 2014. Precise and Ultrafast Molecular Sieving Through Graphene Oxide Membranes. Science. 343:752-54.

65. Huang LL, Zhang LZ, Shao Q, Wang J, Lu LH, Lu XH, et al. 2006. Molecular dynamics simulation study of the structural characteristics of water molecules confined in functionalized carbon nanotubes. J Phys Chem B. 110:25761-68. 
66. Diallo SO, Vlcek L, Mamontov E, Keum JK, Chen JH, Hayes JS, et al. 2015. Translational diffusion of water inside hydrophobic carbon micropores studied by neutron spectroscopy and molecular dynamics simulation. Phys Rev E. 91:022124.

67. Marti J, Gordillo MC. 2003. Structure and dynamics of liquid water adsorbed on the external walls of carbon nanotubes. J Chem Phys. 119:12540-46.

68. Striolo A, Chialvo AA, Cummings PT, Gubbins KE. 2003. Water adsorption in carbon-slit nanopores. Langmuir. 19:8583-91.

69. Striolo A, Gubbins KE, Gruszkiewicz MS, Cole DR, Simonson JM, Chialvo AA. 2005. Effect of temperature on the adsorption of water in porous carbons. Langmuir. 21:9457-67.

70. Chandler D. 2005. Interfaces and the driving force of hydrophobic assembly. Nature. 437:640-47.

71. Kauzmann W. 1959. Some Factors in the Interpretation of Protein Denaturation. Adv Protein Chem. 14:1-63.

72. Stillinger FH. 1973. Structure in Aqueous Solutions of Nonpolar Solutes from the Standpoint of Scaled-Particle Theory. J Solution Chem. 2:141-58.

73. Lum K, Chandler D, Weeks JD. 1999. Hydrophobicity at small and large length scales. J Phys Chem B. 103:4570-77.

74. Huang X, Margulis CJ, Berne BJ. 2003. Dewetting-induced collapse of hydrophobic particles. P Natl Acad Sci USA. 100:11953-58.

75. Cerdeirina CA, Debenedetti PG, Rossky PJ, Giovambattista N. 2011. Evaporation Length Scales of Confined Water and Some Common Organic Liquids. J Phys Chem Lett. 2:1000-03.

76. Altabet YE, Debenedetti PG. 2014. The role of material flexibility on the drying transition of water between hydrophobic objects: A thermodynamic analysis. JChem Phys. 141:18C531.

77. Remsing RC, Xi E, Vembanur S, Sharma S, Debenedetti PG, Garde S, et al. 2015. Pathways to dewetting in hydrophobic confinement. P Natl Acad Sci USA. 112:8181-86.

78. Bakli C, Chakraborty S. 2013. Effect of presence of salt on the dynamics of water in uncharged nanochannels. J Chem Phys. 138:054504.

79. Barrat JL, Bocquet L. 1999. Influence of wetting properties on hydrodynamic boundary conditions at a fluid/solid interface. Faraday Discuss. 112:119-27.

80. Bakli C, Chakraborty S. 2015. Electrokinetic energy conversion in nanofluidic channels: Addressing the loose ends in nanodevice efficiency. Electrophoresis. 36:675-81.

81. Joly L, Ybert C, Trizac E, Bocquet L. 2006. Liquid friction on charged surfaces: From hydrodynamic slippage to electrokinetics. J Chem Phys. 125:204716.

82. Ho TA, Papavassiliou DV, Lee LL, Striolo A. 2011. Liquid water can slip on a hydrophilic surface. P Natl Acad Sci USA. 108:16170-75.

83. Tocci G, Joly L, Michaelides A. 2014. Friction of Water on Graphene and Hexagonal Boron Nitride from Ab Initio Methods: Very Different Slippage Despite Very Similar Interface Structures. Nano Lett. 14:6872-77.

84. Bocquet L, Barrat JL. 1994. Hydrodynamic Boundary-Conditions, CorrelationFunctions, and Kubo Relations for Confined Fluids. Phys Rev E. 49:3079-92. 
85. Bocquet L, Barrat JL. 2013. On the Green-Kubo relationship for the liquidsolid friction coefficient. J Chem Phys. 139:044704.

86. Willard AP, Chandler D. 2009. Coarse-grained modeling of the interface between water and heterogeneous surfaces. Faraday Discuss. 141:209-20.

87. Acharya H, Vembanur S, Jamadagni SN, Garde S. 2010. Mapping hydrophobicity at the nanoscale: Applications to heterogeneous surfaces and proteins. Faraday Discuss. 146:353-65.

88. Giovambattista N, Lopez CF, Rossky PJ, Debenedetti PG. 2008. Hydrophobicity of protein surfaces: Separating geometry from chemistry. P Natl Acad Sci USA. 105:2274-79.

89. Giovambattista N, Rossky PJ, Debenedetti PG. 2009. Effect of Temperature on the Structure and Phase Behavior of Water Confined by Hydrophobic, Hydrophilic, and Heterogeneous Surfaces. J Phys Chem B. 113:13723-34.

90. Hua L, Zangi R, Berne BJ. 2009. Hydrophobic Interactions and Dewetting between Plates with Hydrophobic and Hydrophilic Domains. J Phys Chem C. 113:5244-53.

91. Gadaleta A, Biance AL, Siria A, Bocquet L. 2015. Ultra-sensitive flow measurement in individual nanopores through pressure - driven particle translocation. Nanoscale. 7:7965-70.

92. Lee CY, Choi W, Han JH, Strano MS. 2010. Coherence Resonance in a SingleWalled Carbon Nanotube Ion Channel. Science. 329:1320-24.

93. Christenson HK. 2001. Confinement effects on freezing and melting. J PhysCondens Mat. 13:R95-R133.

94. Majumder M, Zhan X, Andrews R, Hinds BJ. 2007. Voltage gated carbon nanotube membranes. Langmuir. 23:8624-31.

95. Reina A, Thiele S, Jia XT, Bhaviripudi S, Dresselhaus MS, Schaefer JA, et al. 2009. Growth of Large-Area Single- and Bi-Layer Graphene by Controlled Carbon Precipitation on Polycrystalline Ni Surfaces. Nano Res. 2:509-16.

96. Reina A, Jia XT, Ho J, Nezich D, Son HB, Bulovic V, et al. 2009. Large Area, Few-Layer Graphene Films on Arbitrary Substrates by Chemical Vapor Deposition. Nano Lett. 9:30-35.

97. Werder T, Walther JH, Jaffe RL, Halicioglu T, Koumoutsakos P. 2003. On the Water-Carbon Interaction for Use in Molecular DynamicsSimulations of Graphite and Carbon Nanotubes. J Phys Chem B. 107:1345-52.

98. Hall JE. 1975. Access Resistance of a Small Circular Pore. J Gen Physiol. 66:531-32.

99. Greenlee LF, Lawler DF, Freeman BD, Marrot B, Moulin P. 2009. Reverse osmosis desalination: Water sources, technology, and today's challenges. Water Res. 43:2317-48.

100. Busch M, Mickols WE. 2004. Reducing energy consumption in seawater desalination. Desalination. 165:299-312.

101. Lin SH, Elimelech M. 2015. Staged reverse osmosis operation: Configurations, energy efficiency, and application potential. Desalination. 366:9-14.

102. Cohen-Tanugi D, McGovern RK, Dave SH, Lienhard JH, Grossman JC. 2014. Quantifying the potential of ultra-permeable membranes for water desalination. Energ Environ Sci. 7:1134-41. 
103. Gu MH, Vegas AJ, Anderson DG, Langer RS, Kilduff JE, Belfort G. 2013. Combinatorial synthesis with high throughput discovery of protein-resistant membrane surfaces. Biomaterials. 34:6133-38.

104. Imbrogno J, Williams MD, Belfort G. 2015. A New Combinatorial Method for Synthesizing, Screening, and Discovering Antifouling Surface Chemistries. Acs Appl Mater Inter. 7:2385-92.

105. Hansen CM. Hansen Solubility Parameters: A User's Handbook. 2nd Ed. ed. Taylor and Francis Group, LLC: Boca Raton, FL; 2007.

106. Kwan SE, Bar-Zeev E, Elimelech M. 2015. Biofouling in forward osmosis and reverse osmosis: Measurements and mechanisms. J Membrane Sci. 493:703-08.

107. Striolo A. 2011. From Interfacial Water to Macroscopic Observables: A Review. Adsorpt Sci Technol. 29:211-58.

108. Striolo A. 2014. Understanding interfacial water and its role in practical applications using molecular simulations. Mrs Bull. 39:1062-68.

109. Patel AJ, Varilly P, Jamadagni SN, Acharya H, Garde S, Chandler D. 2011. Extended surfaces modulate hydrophobic interactions of neighboring solutes. P Natl Acad Sci USA. 108:17678-83.

110. Phan A, Cole DR, Striolo A. 2014. Aqueous Methane in Slit-Shaped Silica Nanopores: High Solubility and Traces of Hydrates. J Phys Chem C. 118:4860-68.

111. Oren Y. 2008. Capacitive delonization (CDI) for desalination and water treatment - past, present and future (a review). Desalination. 228:10-29.

112. Anderson MA, Cudero AL, Palma J. 2010. Capacitive deionization as an electrochemical means of saving energy and delivering clean water. Comparison to present desalination practices: Will it compete? Electrochim Acta. 55:3845-56.

113. Chmiola J, Yushin G, Gogotsi Y, Portet C, Simon P, Taberna PL. 2006. Anomalous increase in carbon capacitance at pore sizes less than 1 nanometer. Science. 313:1760-63.

114. Winter M, Brodd RJ. 2004. What are batteries, fuel cells, and supercapacitors? Chem Rev. 104:4245-69.

115. Zhang LL, Zhao XS. 2009. Carbon-based materials as supercapacitor electrodes. Chem Soc Rev. 38:2520-31.

116. Simon P, Gogotsi Y. 2008. Materials for electrochemical capacitors. Nat Mater. 7:845-54.

117. Suss ME, Baumann TF, Bourcier WL, Spadaccini CM, Rose KA, Santiago JG, et al. 2012. Capacitive desalination with flow-through electrodes. Energ Environ Sci. 5:9511-19.

118. Porada S, Zhao R, van der Wal A, Presser V, Biesheuvel PM. 2013. Review on the science and technology of water desalination by capacitive deionization. Prog Mater Sci. 58:1388-442.

119. Porada S, Sales BB, Hamelers HVM, Biesheuvel PM. 2012. Water Desalination with Wires. J Phys Chem Lett. 3:1613-18.

120. Feng GA, Qiao R, Huang JS, Sumpter BG, Meunier V. 2010. Atomistic Insight on the Charging Energetics in Subnanometer Pore Supercapacitors. J Phys Chem C. 114:18012-16.

121. Yang L, Garde S. 2007. Modeling the selective partitioning of cations into negatively charged nanopores in water. J Chem Phys. 126:084706. 
122. Shim Y, Kim HJ. 2010. Nanoporous Carbon Supercapacitors in an Ionic Liquid: A Computer Simulation Study. Acs Nano. 4:2345-55.

123. Kalluri RK, Konatham D, Striolo A. 2011. Aqueous NaCl Solutions within Charged Carbon-Slit Pores: Partition Coefficients and Density Distributions from Molecular Dynamics Simulations. J Phys Chem C. 115:13786-95.

124. Chialvo AA, Cummings PT. 2011. Aqua Ions-Graphene Interfacial and Confinement Behavior: Insights from Isobaric-Isothermal Molecular Dynamics. J Phys Chem A. 115:5918-27.

125. Fedorov MV, Kornyshev AA. 2008. Towards understanding the structure and capacitance of electrical double layer in ionic liquids. Electrochim Acta. 53:6835-40.

126. Kalluri RK, Biener MM, Suss ME, Merrill MD, Stadermann M, Santiago JG, et al. 2013. Unraveling the potential and pore-size dependent capacitance of slit-shaped graphitic carbon pores in aqueous electrolytes. Phys Chem Chem Phys. 15:2309-20.

127. Kalluri RK, Ho TA, Biener J, Biener MM, Striolo A. 2013. Partition and Structure of Aqueous $\mathrm{NaCl}$ and $\mathrm{CaCl} 2$ Electrolytes in Carbon-Slit Electrodes. J Phys Chem C. 117:13609-19.

128. Ho TA, Striolo A. 2013. Capacitance enhancement via electrode patterning. $J$ Chem Phys. 139:204708.

129. Ho TA, Striolo A. 2015. Promising Performance Indicators for Water Desalination and Aqueous Capacitors Obtained by Engineering the Electric Double Layer in Nano-Structured Carbon Electrodes. J Phys Chem C. 119:3331-37.

130. Kim S, Lee JK, Kang SO, Ko J, Yum JH, Fantacci S, et al. 2006. Molecular engineering of organic sensitizers for solar cell applications. J Am Chem Soc. 128:16701-07.

131. Ceder G, Chiang YM, Sadoway DR, Aydinol MK, Jang YI, Huang B. 1998. Identification of cathode materials for lithium batteries guided by first-principles calculations. Nature. 392:694-96.

132. Greeley J, Mavrikakis M. 2004. Alloy catalysts designed from first principles. Nat Mater. 3:810-15.

133. Studt F, Abild-Pedersen F, Bligaard T, Sorensen RZ, Christensen CH, Norskov JK. 2008. Identification of non-precious metal alloy catalysts for selective hydrogenation of acetylene. Science. 320:1320-22.

134. Ma J, Michaelides A, Alfe D, Schimka L, Kresse G, Wang EG. 2011. Adsorption and diffusion of water on graphene from first principles. Phys Rev B. 84:033402.

135. Vega C, Abascal JLF, Conde MM, Aragones JL. 2009. What ice can teach us about water interactions: a critical comparison of the performance of different water models. Faraday Discuss. 141:251-76.

136. Babin V, Medders GR, Paesani F. 2012. Toward a Universal Water Model: First Principles Simulations from the Dimer to the Liquid Phase. J Phys Chem Lett. 3:3765-69.

137. Ho TA, Striolo A. 2014. Molecular dynamics simulation of the graphene-water interface: comparing water models. Mol Simulat. 40:1190-200.

138. Liu L, Patey GN. 2014. Simulations of water transport through carbon nanotubes: How different water models influence the conduction rate. J Chem Phys. 141:18C518. 
139. Jungwirth P, Tobias DJ. 2002. Ions at the air/water interface. J Phys Chem B. 106:6361-73.

140. Ho TA, Striolo A. 2013. Polarizability effects in molecular dynamics simulations of the graphene-water interface. J Chem Phys. 138:054117.

141. Ma M, Grey F, Shen LM, Urbakh M, Wu S, Liu JZ, et al. 2015. Water transport inside carbon nanotubes mediated by phonon-induced oscillating friction. Nat Nanotechnol. 10:692-95.

142. Ma M, Tocci G, Michaelides A, Aeppli G. 2015. Fast diffusion of water nanodroplets on graphene. Nat Mater.

143. Carrasco J, Hodgson A, Michaelides A. 2012. A molecular perspective of water at metal interfaces. Nat Mater. 11:667-74.

144. Rafiee J, Mi X, Gullapalli H, Thomas AV, Yavari F, Shi YF, et al. 2012. Wetting transparency of graphene. Nat Mater. 11:217-22.

145. Li ZT, Wang YJ, Kozbial A, Shenoy G, Zhou F, McGinley R, et al. 2013. Effect of airborne contaminants on the wettability of supported graphene and graphite. Nat Mater. 12:925-31.

146. Raj R, Maroo SC, Wang EN. 2013. Wettability of Graphene. Nano Lett. 13:1509-15.

147. Chen J, Li XZ, Zhang QF, Michaelides A, Wang EG. 2013. Nature of proton transport in a water-filled carbon nanotube and in liquid water. Phys Chem Chem Phys. 15:6344-49.

148. Partovi-Azar1 P, Kühne TD. 2015. Many-body dispersion interactions for periodic systems based on maximally localized Wannier functions: Application to graphene/water systems. Phys Status Solidi B

149. Cicero G, Grossman JC, Schwegler E, Gygi F, Galli G. 2008. Water confined in nanotubes and between graphene sheets: A first principle study. J Am Chem Soc. 130:1871-78.

150. Li X, Feng J, Wang EG, Meng S, Klimes J, Michaelides A. 2012. Influence of water on the electronic structure of metal-supported graphene: Insights from van der Waals density functional theory. Phys Rev B. 85:085425.

151. Hamada I. 2012. Adsorption of water on graphene: A van der Waals density functional study. Phys Rev B. 86:195436.

152. Silvestrelli PL, Ambrosetti A. 2014. Including screening in van der Waals corrected density functional theory calculations: The case of atoms and small molecules physisorbed on graphene. J Chem Phys. 140:124107.

153. McKenzie S, Kang HC. 2014. Squeezing water clusters between graphene sheets: energetics, structure, and intermolecular interactions. Phys Chem Chem Phys. 16:26004-15.

154. Burke K. 2012. Perspective on density functional theory. J Chem Phys. 136:150901.

155. Becke AD. 2014. Perspective: Fifty years of density-functional theory in chemical physics. J Chem Phys. 140:18A301.

156. Klimes J, Michaelides A. 2012. Perspective: Advances and challenges in treating van der Waals dispersion forces in density functional theory. J Chem Phys. 137:120901. 
157. Wu YB, Aluru NR. 2013. Graphitic Carbon-Water Nonbonded Interaction Parameters. J Phys Chem B. 117:8802-13.

158. Jenness GR, Karalti O, Jordan KD. 2010. Benchmark calculations of wateracene interaction energies: Extrapolation to the water-graphene limit and assessment of dispersion-corrected DFT methods. Phys Chem Chem Phys. 12:637581.

159. Jenness GR, Karalti O, Al-Saidi WA, Jordan KD. 2011. Evaluation of Theoretical Approaches for Describing the Interaction of Water with Linear Acenes. J Phys Chem A. 115:5955-64.

160. Jenness GR, Jordan KD. 2009. DF-DFT-SAPT Investigation of the Interaction of a Water Molecule to Coronene and Dodecabenzocoronene: Implications for the Water-Graphite Interaction. J Phys Chem C. 113:10242-48.

161. Rubes M, Kysilka J, Nachtigall P, Bludsky O. 2010. DFT/CC investigation of physical adsorption on a graphite (0001) surface. Phys Chem Chem Phys. 12:643844.

162. Voloshina E, Usvyat D, Schutz M, Dedkov Y, Paulus B. 2011. On the physisorption of water on graphene: a $\operatorname{CCSD}(\mathrm{T})$ study. Phys Chem Chem Phys. 13:12041-47.

163. Aragones JL, Sanz E, Vega C. 2012. Solubility of $\mathrm{NaCl}$ in water by molecular simulation revisited. J Chem Phys. 136:244505.

164. Mester Z, Panagiotopoulos AZ. 2015. Temperature-dependent solubilities and mean ionic activity coefficients of alkali halides in water from molecular dynamics simulations. J Chem Phys. 143:044505.

165. Ding Y, Hassanali AA, Parrinello M. 2014. Anomalous water diffusion in salt solutions. P Natl Acad Sci USA. 111:3310-15.

166. Kim JS, Wu Z, Morrow AR, Yethiraj A, Yethiraj A. 2012. Self-Diffusion and Viscosity in Electrolyte Solutions. J Phys Chem B. 116:12007-13.

167. Jiang H, Mester Z, Moultos OA, Economou IG, Panagiotopoulos AZ. 2015. Thermodynamic and Transport Properties of $\mathrm{H} 2 \mathrm{O}+\mathrm{NaCl}$ from Polarizable Force Fields. J Chem Theory Comput. 11:3802-10.

168. Kiss PT, Baranyai A. 2014. A new polarizable force field for alkali and halide ions. J Chem Phys. 141:114501.

169. Hassanali AA, Cuny J, Verdolino V, Parrinello M. 2014. Aqueous solutions: state of the art in ab initio molecular dynamics. Philos T R Soc A. 372:20120482.

170. Bankura A, Santrab B, Jr. RAD, Swartz CW, Klein ML, Wu X. 2015. A systematic study of chloride ion solvation in water using van der Waals inclusive hybrid density functional theory. Molecular Physics: An International Journal at the Interface Between Chemistry and Physics. 113:2842-54.

171. Li H, Francisco JS, Zeng XC. 2015. Unraveling the mechanism of selective ion transport in hydrophobic subnanometer channels. P Natl Acad Sci USA. 112:1085156.

172. Liu J, Shi G, Guo P, Yang J, Fang H. 2015. Blockage of water flow in carbon nanotubes by ions due to interactions between cations and aromatic rings. Phys Rev Lett. 115:164502(6).

173. Bussi G, Donadio D, Parrinello M. 2007. Canonical sampling through velocity rescaling. J Chem Phys. 126:014101. 
174. Thomas M, Corry B. 2015. Thermostat choice significantly influences water flow rates in molecular dynamics studies of carbon nanotubes. Microfluid Nanofluid. 18:41-47.

175. Merlet C, Rotenberg B, Madden PA, Taberna PL, Simon P, Gogotsi Y, et al. 2012. On the molecular origin of supercapacitance in nanoporous carbon electrodes. Nat Mater. 11:306-10.

176. Merlet C, Pean C, Rotenberg B, Madden PA, Simon P, Salanne M. 2013. Simulating Supercapacitors: Can We Model Electrodes As Constant Charge Surfaces? J Phys Chem Lett. 4:264-68.

177. Palmer JC, Gubbins KE. 2012. Atomistic models for disordered nanoporous carbons using reactive force fields. Micropor Mesopor Mat. 154:24-37.

178. Jain SK, Pellenq RJM, Pikunic JP, Gubbins KE. 2006. Molecular modeling of porous carbons using the hybrid reverse Monte Carlo method. Langmuir. 22:994248.

179. Palmer JC, Llobet A, Yeon SH, Fischer JE, Shi Y, Gogotsi Y, et al. 2010. Modeling the structural evolution of carbide-derived carbons using quenched molecular dynamics. Carbon. 48:1116-23.

180. Angelikopoulos P, Papadimitriou C, Koumoutsakos P. 2012. Bayesian uncertainty quantification and propagation in molecular dynamics simulations: A high performance computing framework. J Chem Phys. 137:144103.

181. Behler J. 2015. Constructing high-dimensional neural network potentials: A tutorial review. Int J Quantum Chem. 115:1032-50.

182. Bartok AP, Csanyi G. 2015. Gaussian approximation potentials: A brief tutorial introduction. Int J Quantum Chem. 115:1051-57.

183. Heiranian M, Farimani AB, Aluru NR. 2015. Water desalination with a singlelayer $\mathrm{MoS}_{2}$ nanopore. Nat Commun. 6:8616. 